\title{
The augment of regulatory $T$ cells undermines the efficacy of anti-PD-L1 treatment in cervical cancer
}

Fengying $\mathrm{Xu}^{1 \dagger}$, Fengying Zhang ${ }^{1 \dagger}$, Qian Wang ${ }^{2}$, Ying $X u^{1}$, Shuifang $X u^{1}$, Caihong Zhang ${ }^{1}$ and Lihua Wang ${ }^{3 *}$

\begin{abstract}
Background: Immune checkpoint inhibitors have aroused great expectation of tumor eradication. However, the effect of anti-PD-L1 treatment for cervical cancer is unsatisfactory and the underlying antagonist to anti-PD-L1 efficacy is remained to be studied. Here, we investigated the anti-tumor effect of anti-PD-L1 treatment in cervical tumor model and identified the antagonist to the therapeutic efficacy of anti-PD-L1 treatment.

Results: We found that PD-L1 exhibited a moderate expression in both cervical tumor cell lines and clinical samples compared to other tumor types and the para-tumor tissue respectively. Interestingly, our results showed that the antiPD-L1 treated mice were dichotomously divided into responsive and unresponsive group after five cycles of anti-PDL1 treatment although all the mice had the same genome background. In addition, the unresponsive tumors showed less tumor necrosis area and higher immunosuppression activity induced by regulatory $T$ cells (Tregs) population than the responsive ones. Furthermore, we found that anti-PD-L1 treatment autonomously upregulated Tregs proliferation and frequency in multiple immune organs, and, most importantly, Tregs depletion significantly depressed the tumor growth rate and tumor weight compared with either anti-PD-L1 or anti-CD25 treatment alone. Finally, we observed that the upregulating effector $C D 8^{+} \mathrm{T}$ cell is associated with the better therapeutic effect of anti-PD-L1 therapy post Tregs depletion.
\end{abstract}

Conclusions: Anti-PD-L1 treatment upregulates Tregs frequency and proliferation in tumor model, and the depletion of Tregs may be a useful adjuvant strategy for anti-PD-L1 therapy of cervical cancer.

\section{Introduction}

Cervical cancer, as the second most malignant gynecological tumor with high incidence and high mortality among women, severely threats women's health all around the world $[1,2]$. One of the most important reasons for the increasing trend of cervical cancer is the higher frequency exposure to human papillomavirus (HPV) caused by the bad sexual habits, such as early beginning of sexual

\footnotetext{
*Correspondence: drwanglh0420@163.com
}

${ }^{\dagger}$ Fengying $\mathrm{Xu}$ and Fengying Zhang have contributed equally to this work ${ }^{3}$ Department of Gynecologic Oncology, International Peace Maternity \& Child Health Hospital, Shanghai JiaoTong University School of Medicine, 910 Hengshan Road, Xuhui District, Shanghai 200030, China

Full list of author information is available at the end of the article activities and multiple partners [3]. Currently, the main therapy for cervical cancer includes radiotherapy, chemotherapy, surgery and targeted therapy [4]. However, both radiotherapy and chemotherapy caused serious sideeffects, such as hair loss, nausea, anorexia and diarrhea, which undermines the quality of patient life. Besides, the common total radical hysterectomy and bilateral pelvic lymphadenectomy surgery may by futile for patients with metastasis or at advance stage [5]. Although EGFR and COX-2 mediated targeted therapy have been used for the treatment of cervical cancer, the survival rate and prognosis of cervical patients were not significantly improved $[6,7]$.

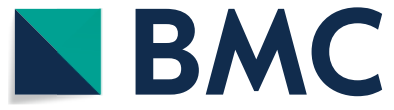

(c) The Author(s) 2021. Open Access This article is licensed under a Creative Commons Attribution 4.0 International License, which permits use, sharing, adaptation, distribution and reproduction in any medium or format, as long as you give appropriate credit to the original author(s) and the source, provide a link to the Creative Commons licence, and indicate if changes were made. The images or other third party material in this article are included in the article's Creative Commons licence, unless indicated otherwise in a credit line to the material. If material is not included in the article's Creative Commons licence and your intended use is not permitted by statutory regulation or exceeds the permitted use, you will need to obtain permission directly from the copyright holder. To view a copy of this licence, visit http://creativecommons.org/licenses/by/4.0/. The Creative Commons Public Domain Dedication waiver (http://creativeco mmons.org/publicdomain/zero/1.0/) applies to the data made available in this article, unless otherwise stated in a credit line to the data. 
The initiation and progression of cervical cancer are associated with the immunosuppression on $\mathrm{CD}_{4}^{+}$and $\mathrm{CD}^{+} \mathrm{T}$ cells caused by HPV infection. Recently, immune checkpoint inhibitors, such as PD-1, PD-L1 and CTLA4, have been intensely studied in many solid tumors, and many clinical trials have shown the long-lasting improved prognosis of patients, especially in melanoma and lung cancer [8-10]. As for cervical cancer, antiCTLA-4 showed little clinical efficacy in patients with recurrence or metastasis [11]. Previous studies showed that tumoral PD-L1 expression was observed in $72 \%$ cervical and vulvar squamous carcinomas (SCC) and 95\% cervical intraepithelial neoplasias (CINs) [9, 12]. Meanwhile, Meng et al. reported that $61 \%$ (59/97) of the patients exhibited PD-1 expression in the tumor stroma of cervical cancer [13]. Pembrolizumab (anti-PD-1) also had been approved by FDA for advanced cervical cancer, and the clinical studies have demonstrated that pembrolizumab demonstrated antitumor activity and exhibited a safety profile in patients with programmed death ligand 1-positive advanced cervical cancer $[14,15]$. Even so, the overall response rate was only $14.3-17 \%$, and $75 \%$ patients experienced treatment related adverse events, such as rash and pyrexia $[14,16]$. Therefore, the therapeutic efficiency of anti-PD-L1 treatment is urgent to be improved in advanced cervical cancer.

Regulatory $\mathrm{T}$ cells (Tregs), defined by $\mathrm{CD}^{+}{ }^{+} \mathrm{CD} 4{ }^{+} \mathrm{CD} 25^{-} \mathrm{FOXP}^{+}$, played an important role in immune escape and thus undermined the therapeutic efficacy of immunotherapy in various tumor types. Previous studies showed that TNFR2 ${ }^{+}$Tregs increased in tumors of cervical cancer patients, and Foxp $3^{+}$tumor infiltrating immune cells in the central tumor area might be a biomarker for risk stratification in cervical cancer patients [17-19]. In contrast, Simone Punt et al. reported that a high total number of Tregs were significantly correlated with improved disease-specific and disease-free survival in cervical adenocarcinoma [20]. However, the effect of anti-PD-L1 on Tregs levels and functions is not clear in cervical cancer. Here, we explore the effect of PD-L1 treatment in syngeneic cervical tumor model and investigated the potential role of Tregs in undermining the effect of anti-PD-L1 therapy in cervical cancer.

\section{Methods}

\section{Cells and regents}

U14 and Hela cell lines were purchased from ATCC, and were cultured in DMEM supplemented with $10 \%$ FBS $+1 \%$ penicillin/streptomycin antibiotics.

\section{Patient samples and mouse model}

Six clinical samples of cervical cancer patients were collected from the department of gynaecology and obstetrics, Jinshan district, Tinglin hospital. Written informed consent forms were obtained from all the patients. The study was approved by the Ethics Committee of the Institute of the department of gynaecology and obstetrics, Jinshan district, Tinglin hospital.

The 6-8 weeks age C57BL/6J mouse used for tumor model was bought from Nanjing Model Animal Center in China. All procedures about mice were approved by the Animal Ethics Committee of shanghai Jiaotong University.

\section{Protein extraction and western blot}

For U14 and Hela cell lines, the collected cells were washed by cold PBS for two times. $2 \times 10^{6}$ cells were treated by RAPA buffer on ice for $30 \mathrm{~min}$. Then, the lysate was centrifuged for $15 \mathrm{~min}$ at $12,000 \mathrm{~g}$ at $4{ }^{\circ} \mathrm{C}$. Then, the protein concentration was measured by BCA method. The loading sample was made at the concentration of $1 \mu \mathrm{g} / \mu \mathrm{l}$ and were loaded for $10 \mu \mathrm{l}$ in SDS-PAGE gel and conducted protein transfer with NC membrane. The targets bands were cut and blocked by $5 \%$ skim milk for $1 \mathrm{~h}$ at room temperature. Then, the bands were washed by TBST for 3 times, and were incubated by PD-L1 antibody(ab213524, 1:1000) overnight at $4{ }^{\circ} \mathrm{C}$. The bands were washed by TBST for 3 times, and were incubated by the second antibody for $1 \mathrm{~h}$ at room temperature. Finally, the blotting signal was recorded under machine.

\section{In vivo tumor progression and immunotherapy models}

All the immune competent C57BL/6J SPF mice were purchased from Nanjing model animal center and feed in the facility of Shanghai Jiaotong University. numbered and randomly assigned into different groups. $3 \times 10^{6}$ logarithmic growth phase U14 cells were transplanted subcutaneously into the flanks of 7-week-old C57BL/6 female mice. For the mouse model in Fig. 2, both PBS and anti PD-L1 treatment groups include six mice; for the mouse model in Fig. 4, sixteen mice were equally assigned to PBS, anti-PD-L1, anti-CD25 and anti-PD-L1 plus antiCD25 groups, and all these group were treated with corresponding regents every two days. The tumor size was measured seven days post tumor challenge with a caliper every 2-3 days, and tumor volume was calculated by width $^{2} \times$ length $\times 0.5$. Mice were sacrificed according to the animal welfare requirement at the endpoint (The maximum tumor less than $15 \mathrm{~mm}$ in diameter). The death of mouse during treatment was used as the exclusion criteria.

\section{RNA extraction and RT-PCR}

The cells were harvested and washed for two time with cold PBS. $1 \mathrm{ml}$ Trizol reagent was added in $2 \times 10^{6}$ cells and sufficiently suspended. The total RNA was extract 
according established protocol. In quantitative PCR (q-PCR), the reverse transcription of $1.5 \mu \mathrm{g}$ total RNA were conducted by using SuperScript III First-Strand Synthesis System. The harvested cDNA was diluted for five times by $\mathrm{ddH}_{2} \mathrm{O}$. The SYBR Green PCR Master Mix (Applied Biosystems) was used for qPCR, and three repeats were assigned in a Real-Time PCR System (Applied Biosystems). All used primers for qPCR are listed as follow: mouse-PD-L1: forward-5'- GCT CCAAAGGACTTGTACGTG-3'; reverse-3'-TGA TCTGAAGGGCAGCATTTC-5'; human-PD-L1: forward-5' - GCTGCACTAATTGTCTATTGGGA$3^{\prime}$; reverse-3'-AATTCGCTTGTAGTCGGCACC-5'; mouse-GAPDH: forward-5'-GAAGGTCGGTGTGAA CGGAT-3'; reverse-3'-TGATGGGCTTCCCGTTGA TG-5'; human-GAPDH: forward-5'-CGGATTTGG TCGTATTGGG-3'; reverse-3'-CTCGCTCCTGGA AGATGG-5'; mouse-Ki67: forward-5'-ATCATTGAC CGCTCCTTTAGGT-3'; reverse-3'-GCTCGCCTT GATGGTTCCT-5'. mouse-CD206: forward-5'-CTC TGTTCAGCTATTGGACGC-3'; reverse-3'-CGG AATTTCTGGGATTCAGCTTC-5'; mouse-Ly6G: forward-5'-GAC TTCCTGCAACACAAC TACC-3'; reverse-3' - ACAGCATTACCAGTGATCTCAGT-5'; mouse-Arginase1: forward-5'-TGTCCCTAATGACAG CTCCTT-3; reverse-3'-GGAGCTGTCATTAGGGAC ATCA-5'; mouse-FOXP3: forward-5'-CCCATCCCC AGGAGTCTTG-3'; reverse-3'-ACCATGACTAGG GGCACTGTA-5'; mouse-Ly6c: forward-5'-GCAGTG CTACGAGTGCTATGG-3', reverse-3'-ACTGACGGG TCTTTAGTTTCCTT-5'.

\section{Immunohistochemistry}

Tumor and spleen tissue samples were carefully resected and immediately fixed in $4 \%$ paraformaldehyde overnight at room temperature. The fixed tissues were embedded in standard paraffin wax to product $5-\mu \mathrm{m}$ sections for HE and immunohistochemistry assay. In brief, the tissue sections were deparaffinized in xylene for 3 times (10 $\mathrm{min} /$ time) and rehydrated via an ethanol gradient $(100 \%, 95 \%, 80 \%, 75 \%, 50 \%)$. After antigen retrieval with pH 6.0 citrate buffer, sections were incubated in a $0.3 \%$ $\mathrm{H}_{2} \mathrm{O}_{2}$ solution to remove peroxidase at room temperature for $10 \mathrm{~min}$. Then, the sections were washed by PBS for 3times (10 min/time) and blocked by normal goat serum or $5 \%$ BSA for $1 \mathrm{~h}$ at $37^{\circ} \mathrm{C}$. The sections were then incubated with rat anti-mouse Foxp3 monoclonal antibody (ab215206, 1:200) or anti- PD-L1 (ab213524, 1:200) at $4{ }^{\circ} \mathrm{C}$ overnight. On the second day, the tissue sections were treated with instant SABC kit according to provided protocol. Finally, the sections were stained with hematoxylin and sealed for observation under microscope.

\section{In vivo antibody treatment}

$3 \times 10^{6}$ U14 cells were subcutaneously injected as described above. Seven days post tumor cell injection, anti-PD-L1 antibody [In vivo mab anti-mouse PD-L1 (B7-H1), BioX Cell, BE0101] and anti-CD25 (IL-2R $\alpha$, In vivo plus anti-mouse CD25, BioX Cell, BP0012) were intraperitoneally injected ( $200 \mu \mathrm{g}$ per dose per mouse) as indicated schedule. Mice were euthanized and tumors were harvested after five times' antibody injections. The resected tumors were photographed and measured.

\section{Cell and tissue FACS analysis}

The peripheral blood, tumor, draining lymph node and spleen were isolated from mice. Then, the single cell suspension for these sample were prepared. The single cells suspension was stained with the following antibodies: anti-mouse CD25-BV605, CD3-FITC and CD4-pacific blue were stained for $30 \mathrm{~min}$ at $4{ }^{\circ} \mathrm{C}$, and the samples were treated with fixation/permeabilization solution for $40 \mathrm{~min}$ in dark. Then, the fixed cells were stained by FOXP3-PE and anti-Ki67 for $30 \mathrm{~min}$ at $4{ }^{\circ} \mathrm{C}$. Finally, the samples were washed for two times by cold PBS. For the sorting of Tregs, the staining panel is the same as mentioned above. The isolated cells were resuspended with $1 \mathrm{ml}$ Trizol regent and the total RNA was extract for RT-PCR.

\section{Statistical analysis}

All experiments were repeated three times for statistical analyses. Mice were randomly allocated to experimental and control groups before treatment. Normally distributed data were analyzed by unpaired two-tailed Student's t-test for single comparisons. Two-way ANOVA test was used between groups. A P-value of $<0.05$ was considered statistically significant.

\section{Results}

PD-L1 exhibited high expression in cervical tumor cell lines and tumor tissue

The expression of PD- 1 and PD-L1 are the important predicative biomarker for anti-PD-1/L1 therapeutic efficacy. Therefore, we first investigated the expression of PD-L1 expression in human and mouse cervical cell lines. Our results showed that Hela and U14 cell lines had moderate PD-L1 expression in mRNA (Fig. 1A, C) and protein level (Fig. 1B, D, Additional file 1) compared with other tumor types, indicating the potential therapeutic effect of anti-PD-L1 therapy in cervical cancer. To investigate the expression of PD-1 and PD-L1 expression in cervical patient samples, we conducted immunohistochemistry for PD-1/L1 in the tumor tissue and corresponding para-tumor tissue of cervical patients. We found that 


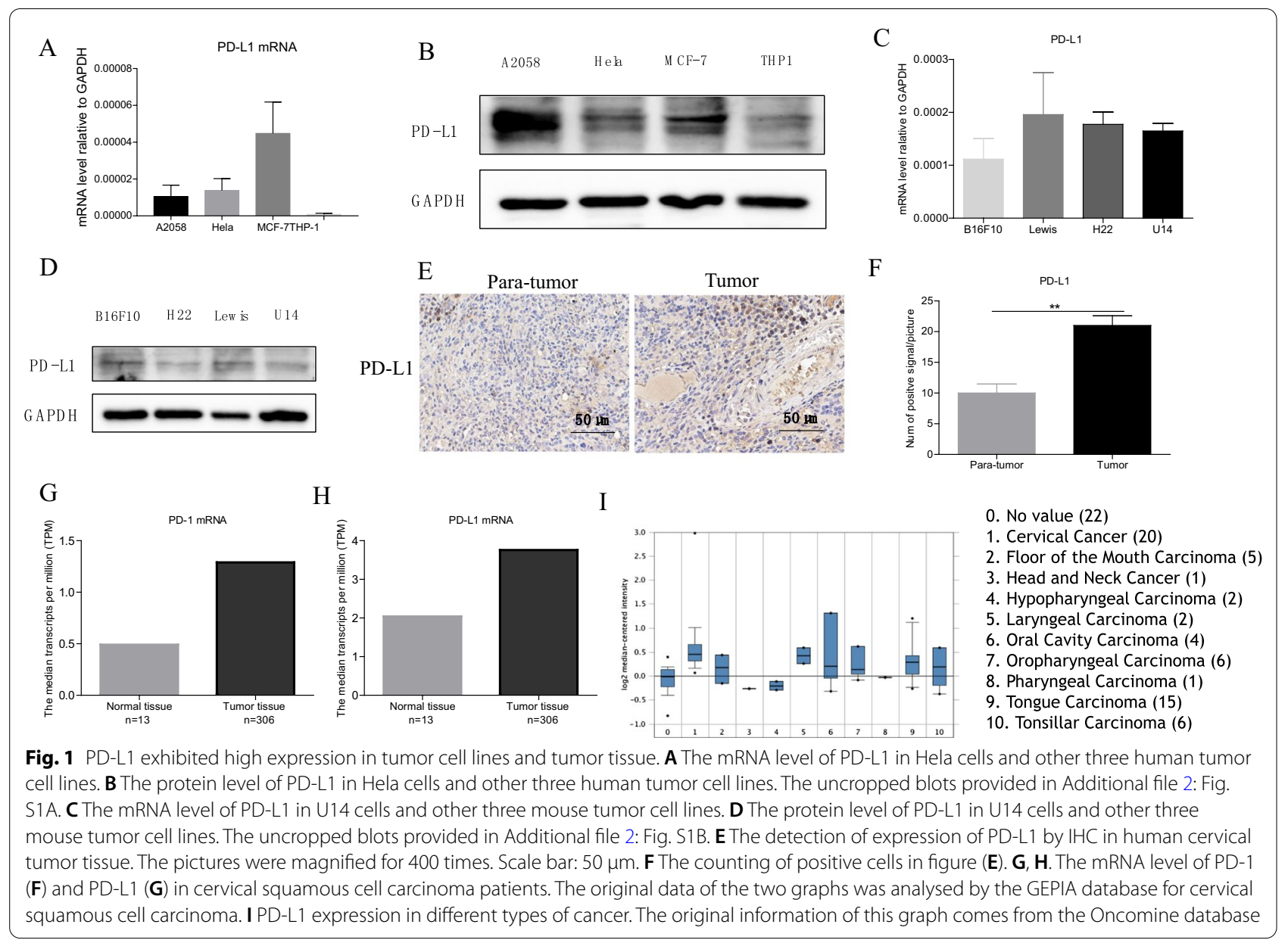

the expression PD-L1 significantly increase in tumor tissue compared to the corresponding para-tumor tissue of patients (Fig. 1E, F). In addition, we analyzed the mRNA level of PD-1 and PD-L1 in human cervical squamous cell carcinoma by GEPIA database and Oncomine database. We observed that PD-1 (Fig. 1G) and PD-L1 (Fig. 1H) showed higher expression in cancer patients than the health controls, and cervical cancer had a relative high PD-L1 expression compared with other common cancer types (Fig. 1I). Taken together, the PD-L1 therapy may be a promising option for the treatment of cervical tumor.

\section{The anti-tumor effect of anti-PD-L1 therapy} was undermined by the enhanced immunosuppression in tumor

To investigate the effect of anti-PD-L1 on tumorigenesis of cervical cancer, we constructed the syngeneic tumor model in immune competent C57BL/6 mouse. Anti-PD-L1 or PBS was administrated according the treatment schedule (Fig. 2A). We found that U14 cell line had $100 \%$ tumor formation rate in $\mathrm{C} 57 \mathrm{BL} / 6$ mice.
Anti-PD-L1 treatment significantly depressed the growth of xenografted tumor in most of mice (Fig. 2B). In contrast, $30 \%$ mice were not response to anti-PDL1 treatment. Then, we conducted HE dye to further investigated the tumor microenvironment situation in responding and non-responding tumors. We found that the tumors responding to anti-PD-L1 treatment showed the higher levels of tumor necrosis than that of unresponsive ones (Fig. 2C). Numerous studies have shown that the immunosuppression activity in tumor microenvironment severely undermined the therapeutic effect of PD-L1 treatment in many types of tumor. Therefore, we extracted mRNA from responsive and unresponsive tumors and detected the immunosuppressive activity by several vital molecules, including Foxp3, CD206, Arginase, Ly6c and Ly6G. Our results showed that the unresponsive tumors showed higher immunosuppressive activity than the responsive one (Fig. 2D). Collectively, the excessive upregulation of Tregs level after anti-PD-L1 treatment may undermine the therapeutic efficiency. 
A

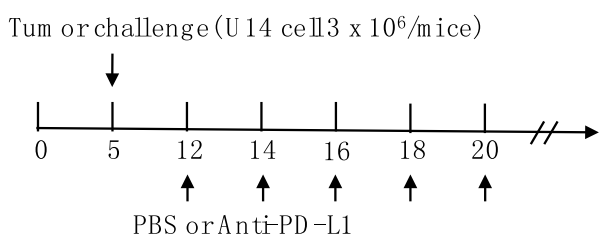

C

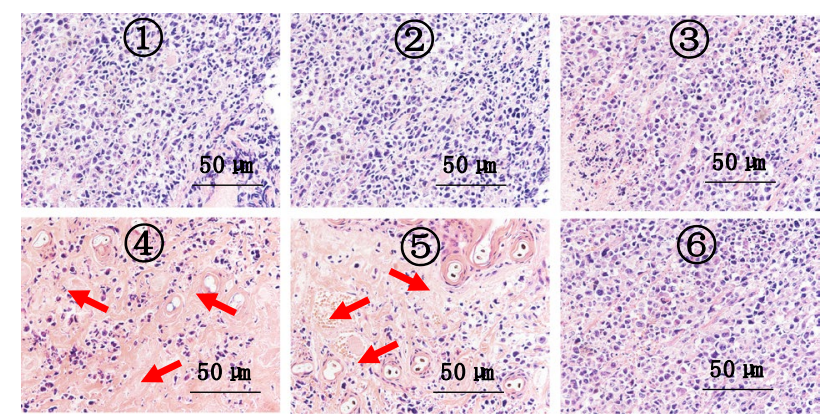

B

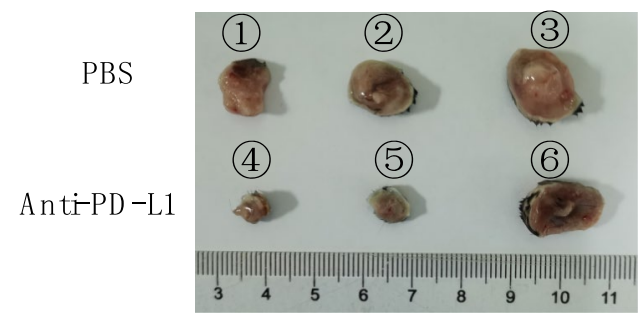

D

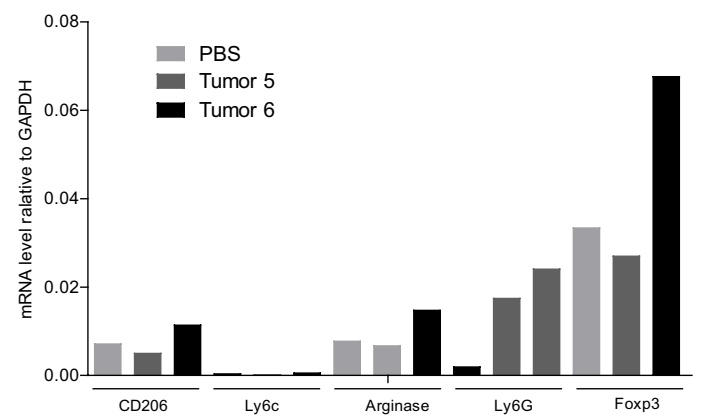

Fig. 2 The anti-tumor effect of anti-PD-L1 treatment was undermined by the enhanced immunosuppression in tumor. A The anti-PD-L1 treatment schedule in syngeneic tumor mouse model. $3 \times 10^{6} \mathrm{U} 14$ cervical cells was subcutaneously injected on the flank of 7-weeks C57BL/6 mouse. Anti-PD-L1 (200 $\mathrm{\mu g} /$ time/mouse) was intraperitoneally injected in mouse seven days post tumor challenge, and five injection were performed. B The representative picture of tumors after five times anti-PD-L1 therapy. The tumor was numbered with Arabic numerals. C The HE dying for the tumors in figure (B), and the red arrow represents the necrosis area. The pictures were magnified for 400 times. Scale bar: $50 \mu m$. D The mRNA level of immunosuppressive molecular, including CD206, Ly6C, Ly6G, Foxp3, and Arginase in control (no PD-L1 treatment), tumor (5) and tumor (6)

The upregulating Tregs in tumors was associated with the compromised therapeutic efficiency of PD-L1 treatment

As shown in the Fig. 2D, the unresponsive tumor showed very high level of Foxp3 compared to the responsive tumor and other immunosuppressive markers. Therefore, we hypothesized that the upregulated Tregs level might account for the compromised anti-tumor effect in unresponsive ones. The IHC results showed that Tregs had a relatively high level in unresponsive tumors (Fig. 3A) and corresponding spleens (Fig. 3B) compared to responsive tumors. To further identified our finding, we conducted the flow cytometry to detect the frequency of Tregs in tumors. Consistently, we indeed observed the highest Tregs frequency in unresponsive tumor (Fig. 3C). Of note, we also found that anti-PD-L1 promoted the frequency of Tregs in both responsive and unresponsive tumors at different degree (Fig. 3C).

Tregs depletion strengthened the anti-tumor effect of anti-PD-L1 treatment in cervical tumor model

Although anti-PD-L1 could effectively depressed the growth rate of tumor in the mouse model, only $20 \%$ reduction of tumor weight was achieved. Therefore, we hypothesized that Tregs depletion could enhance anti-PD-L1 efficacy. Therefore, we used PBS, antiPD-L1, anti-CD25 or anti-PD-L1 plus anti-CD25 to treated cervical tumor mouse model (Additional file 2: Fig. S1A). Then, we performed C-flow cytometry of Tregs population in the various immune organs after several five times immunotherapy (Additional file 2: Fig. S1B). Our results showed that anti-PD-L1 significantly increased the percentage of Tregs in peripheral blood (Fig. 4A), spleen (Fig. 4B), tumors (Fig. 4C) and lymph node (Fig. 4D). Importantly, anti-PD-L1 plus anti-CD25 treatment significantly inhibited the growth of syngeneic tumor compared to PBS or antiPD-L1 or anti-CD25 alone (Fig. 4E). The tumors were harvest at the endpoint, and the tumor weight in antiPD-L1 plus anti-CD25 treatment group was significantly smaller than the control group or anti-PD-L1 or anti-CD25 group alone (Fig. 4F, G). Taken together, Tregs depletion could strengthen the therapeutic effect of anti-PD-L1 treatment by decreasing upregulating immunosuppression after immunotherapy. 


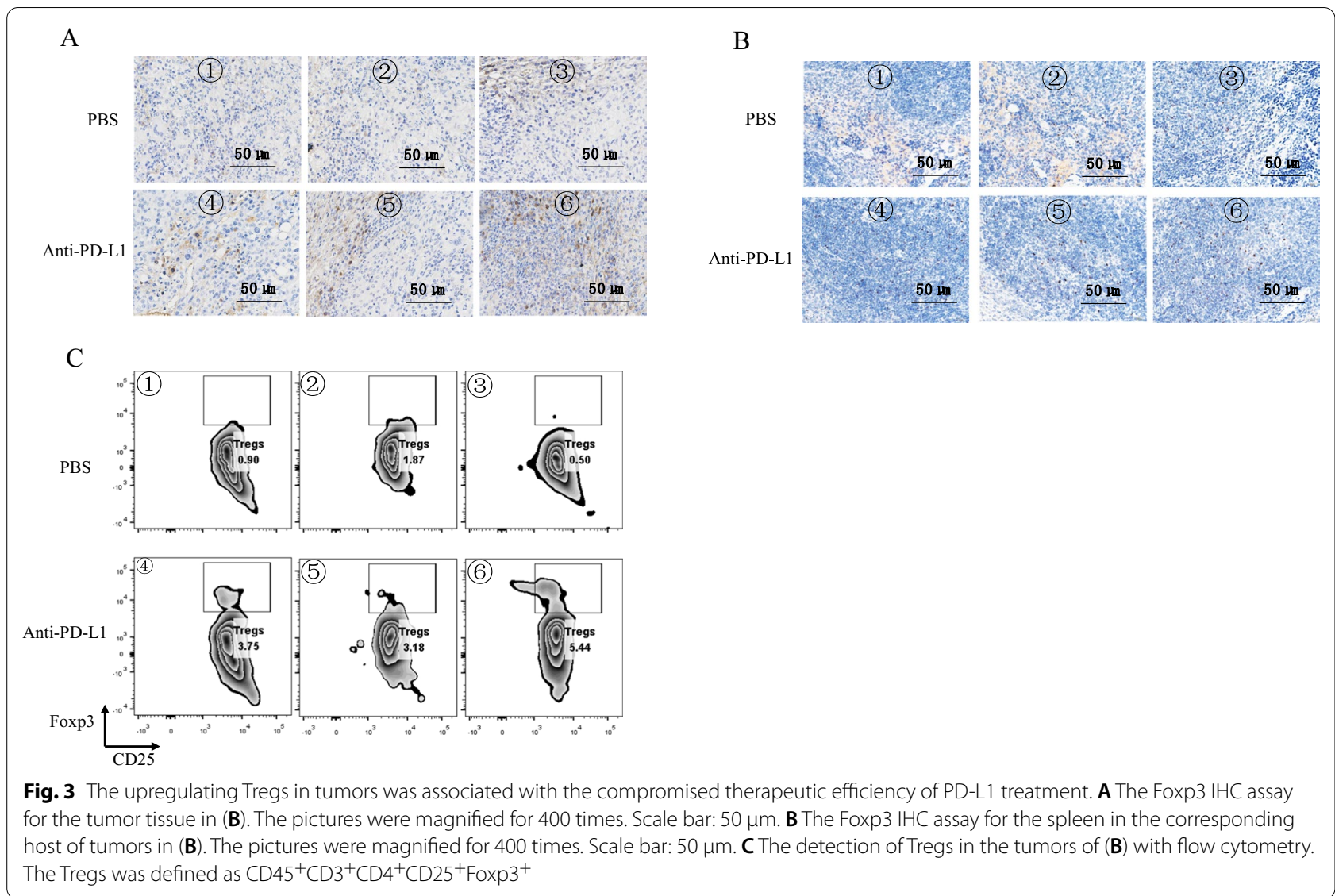

\section{The increased Tregs proliferation depressed the level of effector $\mathrm{CD}^{+} \mathrm{T}$ cells after PD-L1 treatment}

To figure out the reason for the increase of Tregs, we analyzed the signature of Tregs after anti-PD-L1 treatment. We found that anti-PD-L1 treatment significantly upregulated the percentage of $\mathrm{Ki} 7^{+}$Tregs, indicating the increasing Tregs proliferation (Fig. 5A). Additionally, we also sorted Tregs for PBS and PD-L1 treated group to detect the mRNA level of Ki67 transcription. Consistently, Ki67 showed the higher mRNA level in anti-PD-L1 group compared with the PBS group (Fig. 5B). Therefore, the increased Tregs after anti-PD-L1 therapy may associated with increasing proliferation of Tregs.

Considering of the important role of effector $\mathrm{CD}^{+} \mathrm{T}$ cells (defined by $\mathrm{CD}^{+}{ }^{+} \mathrm{CD} 8{ }^{+} \mathrm{CD} 62 \mathrm{~L}^{-} \mathrm{CD} 44^{+}$) in antitumor response. We respectively analyzed the frequency of effector T cells after PBS, or anti-PD-L1 or anti-CD25 or anti-PD-L1 plus anti-CD25 treatment. Our results that anti-PD-L1 plus anti-CD25 treatment group had a significantly higher level of effector $\mathrm{CD} 8^{+} \mathrm{T}$ cells than PBS and PD-L1 group (Fig. 5C, D). In the draining lymph node, we also observed the more distinct increase of effector $\mathrm{CD} 8^{+}$ $\mathrm{T}$ cells in the combination group compared to any of the other three groups (Fig. 5E). In conclusion, the increased effector $\mathrm{CD}^{+} \mathrm{T}$ cells may be associated with the better therapeutic effect after Tregs depletion in cervical tumor model.

\section{Discussion}

Currently, immunotherapy had aroused the widely concern of researchers focusing on various tumor types [21]. Although several clinical trials had verified the effect of anti-PD-L1 on advanced tumors, most of patients had great difficulty in maintaining the long-lasting response to immune checkpoint mediated immunotherapy, let alone the eradicating of tumor cells $[8,11]$. However, the underlying mechanism for the low anti-tumor efficacy of immunotherapy in cervical cancer was not very clear. Here, we found that anti-PD-L1 dichotomously affected tumor growth in the syngeneic mouse model, and the different response to anti-PD-L1 treatment is associated with the autonomously increased Tregs proliferation and frequency in multiple immune organs and tumors. We also found that Tregs depletion significantly enhanced the tumor depression effect of anti-PD-L1 treatment in vivo. Therefore, our research provided a novel insight for the limited anti-tumor efficacy of anti-PD-L1 treatment in cervical cancer. 


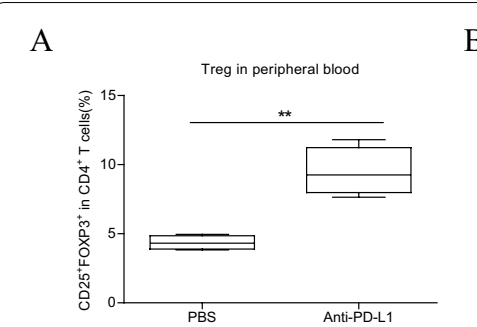

B

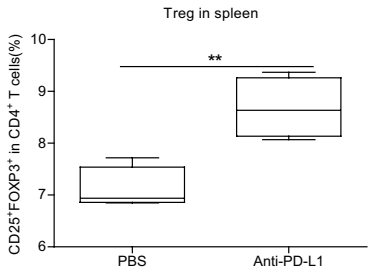

$\mathrm{F}$
$\mathrm{C}$

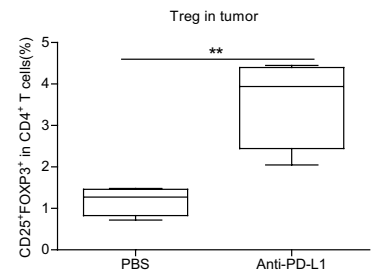

$\mathrm{D}$

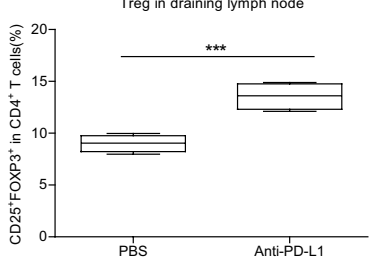

G
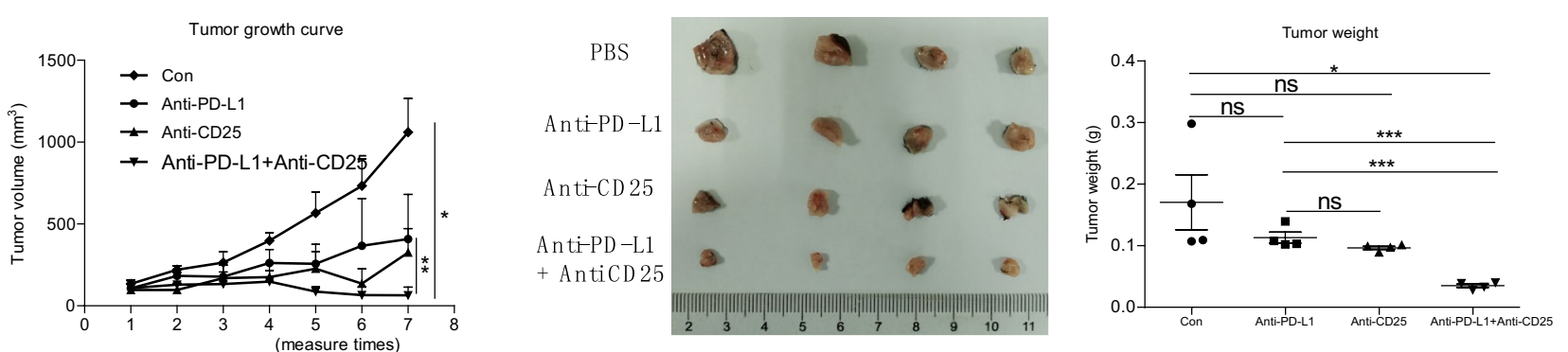

Fig. 4 Tregs depletion strengthened the anti-tumor effect of anti-PD-L1 treatment in cervical tumor model. A-D The change of Tregs level in peripheral blood $(\mathbf{A})$, spleen $(\mathbf{B})$, tumor $(\mathbf{C})$ and $\operatorname{DLN}(\mathbf{D})$ in the syngeneic tumor model. The above samples were collected at the endpoint. $N=4$. Two-tailed unpaired T-test was performed. $\mathbf{E}$ The tumor growth curve under different treatment. The tumor was measured with a caliper, and the tumor volumes were calculated with the formula: $1 / 2 *$ length *width*width. Two-tailed unpaired T-test was performed. $\mathbf{F}$ The tumor pictures after different treatment. $N=4$. $\mathbf{G}$ The tumor weight in different groups. $n=4$. The tumors were weight with a analytical balance. Two-tailed unpaired T-test was performed. ns: no significant difference, ${ }^{*} p<0.05,{ }^{* *} p<0.01$ compared to the control groups. A $p$ value less than 0.05 was considered to be statistically significant

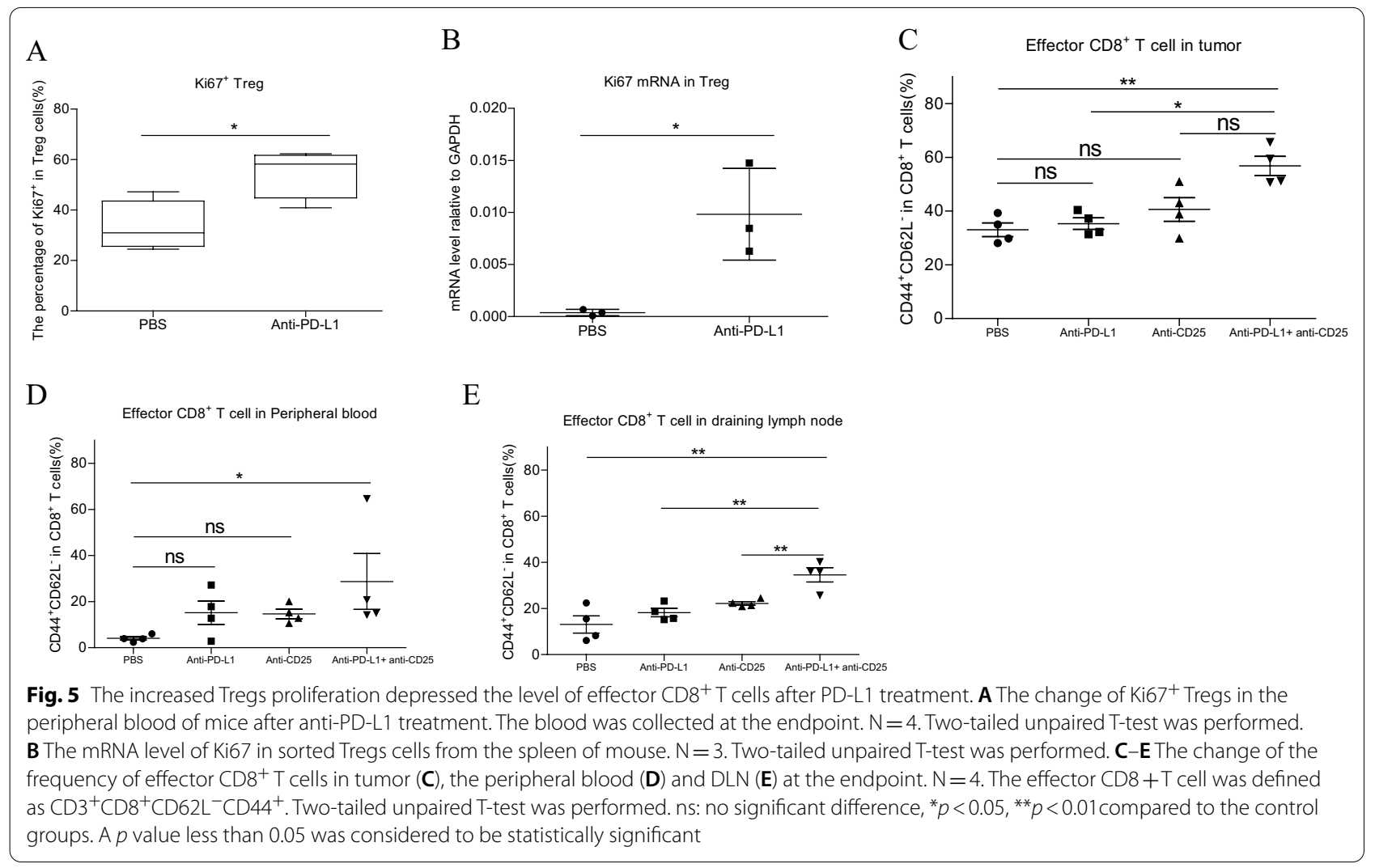


The increased Tregs may be one of the important mechanisms of cervical tumors to resist immunotherapy efficacy. Although we failed to explore the underlying mechanism for Tregs increasing post PD-L1 treatment, we indeed observed the correlation between IL6 expression and Tregs upregulation. Under tumor conditions, various cytokines, such as GM-CSF, IL6, TNF- $\alpha$, and other chemokines [22, 23]. Previous studies showed that IL6 and Tumor necrosis factor $\alpha$ (TNF $\alpha$ ) could promote Tregs proliferation in tumor sites. The recent study reported that anti-PD-L1 treatment or the Rhein plus PD-L1 therapy groups upregulated the IL6 level in the established 4T1 breast cancer xenografts [24]. Consistently, we also observed the slightly increase of IL6 in the tumor tissue after anti-PD-L1 therapy (data not show). TNF is a potent pro-inflammatory cytokine, which played a vital role in the balance of tumor microenvironment. Benoît L Salomon et al. reported that TNF is able to increase expansion, stability, and possibly function of Tregs via TNFR2 [23]. In addition, Lack of interleukin-6 in the tumor microenvironment augments type- $1 \mathrm{immu}$ nity and increases the efficacy of anti-PD-L1 therapy in CT26 cells mouse model [25]. Collectively, we supposed that the increased Tregs probably caused by increased IL6 expression after PD-L1 treatment, which, however, remained to be further confirmed in the future considering of the limited number of mice response to anti-PD-L1 treatment in our project.

Although we observed the enhanced anti-tumor effect after Tregs depletion during anti-PD-L1 treatment in mouse model, we also should be careful for the quickly use of this strategy in clinical cervical cancer patients. A few studies reported that Tregs depleted mice suffered serious autoimmune disease [26]. Furthermore, anti-PD-L1 also may lead to huge immune storm in the host. Therefore, much more attention should be paid on the treatment related adverse event in cervical cancer patients during Tregs depletion combined anti-PDL1 treatment in cervical cancer patients in the future. Admittedly, the animal number in the was a little bit small in this project and more animals might be added in every group to make our conclusion even solid in the future.

In conclusion, we found that anti-PD-L1 treatment upregulated Tregs levels in cervical cancer mouse model, and Tregs depletion maybe a promising adjuvant treatment of anti-PD-L1therapy for cervical cancer treatment.

\section{Supplementary Information}

The online version contains supplementary material available at https://doi. org/10.1186/s12865-021-00451-7.

Additional file 1. The original, uncropped gels for western blot.
Additional file 2. Treatment schedule and the representative figure for Cytometry flow analysis.

Acknowledgements

We are thankful to all members work in the SPF mouse core at Shanghai Jiaotong University

\section{Authors' contributions}

FY Xu and FY Zhang wrote the manuscript. FY Xu, FY Zhang, Q Wang and Y Xu construct the mouse model and collected all related data in Fig. 2-5. FY Xu, FY Zhang, SF Xu and CH Zhang performed the flow cytometry and IHC assay in Fig. 2-5. LH Wang designed and supervised the project. All authors had read and approved the manuscript.

\section{Funding}

This study was supported by grants from Shanghai Jinshan District Science and technology innovation project: No.2020-3-34.

Availability of data and materials

All data and materials were available from the corresponding author.

\section{Declarations}

Competing interests

The authors declare no competing interests.

Ethics approval and consent to participate

All methods were carried out in accordance with relevant guidelines and regulations at Shanghai Jiaotong University. All animal protocols were approved

by Shanghai Jiaotong University Institutional Animal Care and Use Committee.

Consent for publication

All authors have read and approved the publication of the manuscript.

\section{Author details}

'Department of Gynaecology and Obstetrics, Jinshan District Tinglin Hospital, Shanghai 201505, China. ${ }^{2}$ Department of Pathology, Jinshan District Tinglin Hospital, Shanghai 201505, China. ${ }^{3}$ Department of Gynecologic Oncology, International Peace Maternity \& Child Health Hospital, Shanghai JiaoTong University School of Medicine, 910 Hengshan Road, Xuhui District, Shanghai 200030, China.

Received: 19 December 2020 Accepted: 28 August 2021

Published online: 03 September 2021

\section{References}

1. Suh DH, Kim JW, Aziz MF, Devi UK, Ngan HY, Nam JH, Kim SC, Kato T, Ryu HS, Fujii S, et al. Asian society of gynecologic oncology workshop 2010. J Gynecol Oncol. 2010;21(3):137-50.

2. Benard VB, Thomas CC, King J, Massetti GM, Doria-Rose VP, Saraiya M. Centers for Disease C, Prevention: Vital signs: cervical cancer incidence, mortality, and screening - United States, 2007-2012. MMWR Morb Mortal Wkly Rep. 2014;63(44):1004-9.

3. Gustafsson L, Ponten J, Zack M, Adami HO. International incidence rates of invasive cervical cancer after introduction of cytological screening. Cancer Causes Control. 1997;8(5):755-63.

4. Tsikouras P, Zervoudis S, Manav B, Tomara E, latrakis G, Romanidis C, Bothou A, Galazios G. Cervical cancer: screening, diagnosis and staging. J BUON. 2016;21(2):320-5.

5. Kim JY, Byun SJ, Kim YS, Nam JH. Disease courses in patients with residual tumor following concurrent chemoradiotherapy for locally advanced cervical cancer. Gynecol Oncol. 2017;144(1):34-9.

6. Hertlein L, Lenhard M, Kirschenhofer A, Kahlert S, Mayr D, Burges A, Friese K. Cetuximab monotherapy in advanced cervical cancer: a retrospective study with five patients. Arch Gynecol Obstet. 2011;283(1):109-13. 
7. Herrera FG, Chan P, Doll C, Milosevic M, Oza A, Syed A, Pintilie M, Levin W, Manchul L, Fyles A. A prospective phase I-II trial of the cyclooxygenase-2 inhibitor celecoxib in patients with carcinoma of the cervix with biomarker assessment of the tumor microenvironment. Int J Radiat Oncol Biol Phys. 2007;67(1):97-103.

8. Antonia SJ, Villegas A, Daniel D, Vicente D, Murakami S, Hui R, Yokoi T, Chiappori A, Lee KH, de Wit M, et al. Durvalumab after chemoradiotherapy in stage III non-small-cell lung cancer. N Engl J Med. 2017;377(20):1919-29.

9. Mezache L, Paniccia B, Nyinawabera A, Nuovo GJ. Enhanced expression of PD L1 in cervical intraepithelial neoplasia and cervical cancers. Mod Pathol. 2015;28(12):1594-602

10. Hodi FS, O'Day SJ, McDermott DF, Weber RW, Sosman JA, Haanen JB, Gonzalez R, Robert C, Schadendorf D, Hassel JC, et al. Improved survival with ipilimumab in patients with metastatic melanoma. N Engl J Med. 2010;363(8):711-23

11. Lheureux S, Butler MO, Clarke B, Cristea MC, Martin LP, Tonkin K, Fleming GF, Tinker AV, Hirte HW, Tsoref D, et al. Association of ipilimumab with safety and antitumor activity in women with metastatic or recurrent human papillomavirus-related cervical carcinoma. JAMA Oncol. 2018:4(7):e173776.

12. Chinn Z, Stoler MH, Mills AM. PD-L1 and IDO expression in cervical and vulvar invasive and intraepithelial squamous neoplasias: implications for combination immunotherapy. Histopathology. 2019;74(2):256-68.

13. Meng Y, Liang H, Hu J, Liu S, Hao X, Wong MSK, Li X, Hu L. PD-L1 expression correlates with tumor infiltrating lymphocytes and response to neoadjuvant chemotherapy in cervical cancer. J Cancer. 2018;9(16):2938-45.

14. Frenel JS, Le Tourneau C, O'Neil B, Ott PA, Piha-Paul SA, Gomez-Roca C, van Brummelen EMJ, Rugo HS, Thomas S, Saraf S, et al. Safety and efficacy of pembrolizumab in advanced, programmed death ligand 1-positive cervical cancer: results from the phase Ib KEYNOTE-028 trial. J Clin Oncol. 2017;35(36):4035-41.

15. Borcoman E, Le Tourneau C. Pembrolizumab in cervical cancer: latest evidence and clinical usefulness. Ther Adv Med Oncol. 2017:9(6):431-9.

16. Chung HC, Ros W, Delord JP, Perets R, Italiano A, Shapira-Frommer R, Manzuk L, Piha-Paul SA, Xu L, Zeigenfuss S, et al. Efficacy and safety of pembrolizumab in previously treated advanced cervical cancer: results from the phase II KEYNOTE-158 Study. J Clin Oncol. 2019;37(17):1470-8.

17. Adurthi S, Krishna S, Mukherjee G, Bafna UD, Devi U, Jayshree RS. Regulatory T cells in a spectrum of HPV-induced cervical lesions: cervicitis, cervical intraepithelial neoplasia and squamous cell carcinoma. Am J Reprod Immunol. 2008;60(1):55-65.

18. Chen R, Gong Y, Zou D, Wang L, Yuan L, Zhou Q. Correlation between subsets of tumor-infiltrating immune cells and risk stratification in patients with cervical cancer. PeerJ. 2019;7:e7804.

19. Zhang T, Jiao J, Jiao X, Zhao L, Tian X, Zhang Q, Ma D, Cui B. Aberrant frequency of TNFR2 (+) Treg and related cytokines in patients with CIN and cervical cancer. Oncotarget. 2018;9(4):5073-83.

20. Punt S, van Vliet ME, Spaans VM, de Kroon CD, Fleuren GJ, Gorter A, Jordanova ES. FoxP3(+) and IL-17(+) cells are correlated with improved prognosis in cervical adenocarcinoma. Cancer Immunol Immunother. 2015;64(6):745-53

21. Boutros C, Tarhini A, Routier E, Lambotte O, Ladurie FL, Carbonnel F, Izzeddine H, Marabelle A, Champiat S, Berdelou A, et al. Safety profiles of anti-CTLA-4 and anti-PD-1 antibodies alone and in combination. Nat Rev Clin Oncol. 2016;13(8):473-86.

22. Johnson DE, O'Keefe RA, Grandis JR. Targeting the IL-6/JAK/STAT3 signalling axis in cancer. Nat Rev Clin Oncol. 2018;15(4):234-48.

23. Salomon BL, Leclerc M, Tosello J, Ronin E, Piaggio E, Cohen JL. Tumor necrosis factor alpha and regulatory T cells in oncoimmunology. Front Immunol. 2018;9:444.

24. Shen Z, Zhu B, Li J, Qin L. Rhein augments antiproliferative effects of atezolizumab based on breast cancer (4T1) regression. Planta Med. 2019;85(14-15):1143-9.

25. Ohno Y, Toyoshima Y, Yurino H, Monma N, Xiang H, Sumida K, Kaneumi S, Terada S, Hashimoto S, Ikeo K, et al. Lack of interleukin-6 in the tumor microenvironment augments type-1 immunity and increases the efficacy of cancer immunotherapy. Cancer Sci. 2017;108(10):1959-66.

26. Sakaguchi S, Ono M, Setoguchi R, Yagi H, Hori S, Fehervari Z, Shimizu J, Takahashi T, Nomura T. Foxp3+CD25+CD4+ natural regulatory T cells in dominant self-tolerance and autoimmune disease. Immunol Rev. 2006;212:8-27.

\section{Publisher's Note}

Springer Nature remains neutral with regard to jurisdictional claims in published maps and institutional affiliations.
Ready to submit your research? Choose BMC and benefit from:

- fast, convenient online submission

- thorough peer review by experienced researchers in your field

- rapid publication on acceptance

- support for research data, including large and complex data types

- gold Open Access which fosters wider collaboration and increased citations

- maximum visibility for your research: over 100M website views per year

At BMC, research is always in progress.

Learn more biomedcentral.com/submissions 